\title{
Gamificación e interactividade nas aulas da UDC
}

Nieto-Riveiro, Laura; Groba González, Betania; Pereira Loureiro, Javier; Pousada García, Thais

Centro de Investigación en Tecnologías de la Información las Comunicaciones (CITIC)

Facultade de Ciencias da Saúde. Universidade da Coruña.

\section{RESUMO}

Este traballo xestouse no Grupo de Innovación Educativa "Gamificación e interactividade nas aulas" da Universidade da Coruña. Como principal obxectivo planeouse determinar o potencial da creación de contidos interactivos dentro das presentacións de diferentes materias mediante tecnoloxías coma os clickers on-line e ferramentas de gamificación para captar a atención do alumnado. A comunicación presenta os resultados do emprego das enquisas online e en tempo real, a través de diversas aplicacións, coma AnswerGarden, Kahoot e Mentimeter. A metodoloxía interactiva aplicouse en materias do Grao en Terapia Ocupacional e na Universidade Sénior da Universidade da Coruña. Os participantes activos inclúen aos propios docentes, estudantes xoves (entre 19 e 25 anos) e persoas maiores de 50 anos (Universidade Sénior). Os estudantes de $2^{0}$ e $3^{0}$ de Terapia Ocupacional $(n=105)$ participaron nas enquisas (media de 6 consultas online por materia). Na Universidade Sénior, 30 estudantes reflectiron as súas percepcións sobre diferentes temáticas como saúde, benestar e envecellemento e manifestaron os coñecementos acadados sobre 0 uso das TIC. Nesta primeira experiencia, comprobouse que a gamificación pode ser aplicada nos contornos universitarios con diferentes grupos de estudantes. A participación e visualización dos resultados prodúcese en tempo real, ofrecendo unha retroalimentación inmediata e contribuíndo á aprendizaxe de contidos específicos.

PALABRAS CLAVE: Gamificación, Interactividade, Tecnoloxías da Información e as Comunicacións, Participación activa. 


\section{CITA RECOMENDADA:}

Nieto-Riveiro, L.; Groba González, B.; Pereira Loureiro, J. ; Pousada García, T. (2019): Gamificación e interactividade nas aulas da UDC. En De la Torre Fernández, E. (ed.) (2019). Contextos universitarios transformadores: construíndo espazos de aprendizaxe. III Xornadas de Innovación Docente. Cufie. Universidade da Coruña. A Coruña (pág. 71-82).

DOl capítulo: https://doi.org/10.17979/spudc.9788497497121.071

DOl libro: https://doi.org/10.17979/spudc.9788497497121

\section{ABSTRACT}

The present work was created by Group of Educative Innovation "Gamification and interactivity in classrooms" of Universidade da Coruña. The main proposal was to determine the potential derivate from the creation of interactive contains on presentation of few subjects through technologies. One example of that is the use of clickers online and tools for gamification to get the attention from students. The paper presents the results from the use of online and on time enquires through diverse applications, like AnswerGarden, Kahoot and Mentimeter. The interactive methodology was applied in subjects of Degree in Occupational Therapy and of Senior University. The active participants were the own teachers, young students (19-25 years old) and people with more than 50 years (Senior University). The students from 2 and 3 course of Occupational Therapy $(n=105)$ participated on enquires (main of 6 consults by subject). In Senior University, 30 students reflected their perceptions about different subjects, as health, wellbeing and aging, and exposed their own knowledge about ICT. In that first experience, authors checked that gamification can be applied along universities contexts with different groups of students. The participation and display of results occur in real time, offering an immediate feedback and contributing to the knowledge of specific contains.

KEY WORDS: Gamification, Interactivity, Information and Communication Technologies, Active Participation. 


\section{INTRODUCCIÓN}

0 Grupo de Innovación Educativa (GIE) "Gamificación e interactividade nas aulas", xorde no ano 2017, promovido pola respectiva convocatoria para a constitución e recoñecemento dos GIE da Universidade da Coruña (UDC). Un dos obxectivos propostos neste grupo, formado por 15 docentes, foi indagar sobre o potencial da creación de contidos interactivos dentro das presentacións dos temas, mediante tecnoloxías coma os clickers on-line e ferramentas de gamificación para captar a atención do alumnado.

Nesta liña, parte do equipo do GIE aplicou, nas súas actividades docentes, diferentes ferramentas de enquisas en tempo real, incorporando alternativas de comunicación interactiva, que contribuíron co aumento da atención e motivación do estudantado.

A gamificación enténdese coma 0 emprego do xogo e 0 deseño de xogos para a mellora do compromiso e motivación dos participantes. A definición acuñada por Deterding, Khaled, Nacke, \& Dixon (2011) refírese ó "uso de elementos de deseño xogos en contextos que no son de xogo". Neste senso, os compoñentes lúdicos do xogo poden ser aplicados en diversos contextos non vinculados a este, como poden ser os espazos educativos, de traballo ou actividades domésticas como facer a compra. A gamificación conceptualízase coma unha estratexia de márketing, motivación ou fidelización de usuarios (Pérez et al., 2011).

No caso concreto da educación formal, vense empregando a gamificación coma un recurso para potenciar a aprendizaxe, a motivación e 0 compromiso do alumnado coa experiencia (Barbosa \& Soto, 2016).

Segundo Parente (2016), débese ter en conta unha arquitectura funcional no proceso de gamificación no ámbito educativo. Os elementos craves desta arquitectura son (Parente, 2016):

- Actividade: conxunto de accións que estimulan a aprendizaxe e contemplan a resolución de problemas. 
- Contexto: neste estamento tense en conta á propia institución educativa, os seus recursos materiais e tecnolóxicos, a temporalidade, as características da aula e os contornos individuais dos participantes.

- Competencias e habilidades: refírese ás capacidades propias de cada estudante, caracterizadas pola heteroxeneidade. As actividades propostas deben ter en conta esta diversidade para que os resultados non sexan diverxentes en exceso.

- Xestión e supervisión: fai constar a necesidade dunha figura de líder dentro da aula para "deseñar, implementar e controlar a execución do plan de gamificación".

- Mecánicas e elementos dos xogo: dinámicas involucradas nos procesos de aprendizaxe, canalizadas polas actividades da gamificación.

Se ben é certo que a aplicación da gamificación tivo un aumento nos niveis de ensino non universitarios, tamén xa son numerosas as experiencias nas aulas universitarias (Barbosa \& Soto, 2016; Contreras Espinosa \& Eguia, 2016; Pérez et al., 2011). Sen embargo, poucas fan unha análise entre grupos de materias do mesmo título e entre estes e unha poboación maior universitaria.

A continuación, preséntase unha das actividades con maior participación e implicación dos estudantes, e con repercusións positivas sobre a súa motivación, ao tempo que se amosan resultados da gamificación aplicada en grupos estudantes xoves e en persoas maiores.

\section{DESCRICIÓN DA EXPERIENCIA}

Os docentes participantes, da Facultade de Ciencias da Saúde, reuníronse previamente para determinar as formas de aplicación da gamificación nas materias. Establecéronse as ferramentas a empregar e 0 xeito de incluílas como parte integrada e coherente dentro das actividades docentes.

As materias implicadas forman parte do título do Grao en Terapia Ocupacional da Universidade da Coruña (Universidade da Coruña, 2018); así coma a materia "Estimulación cognitiva coas 
TIC" (actualmente denominada "Ferramentas tecnolóxicas para apoiar a saúde e a vida activa") da Universidade Sénior (Universidade da Coruña, 2018b).

No primeiro caso, participaron 105 estudantes dos cursos $2^{\circ}$ e $3^{\circ}$ do Grao en Terapia Ocupacional. Programáronse unha media de 6 consultas online por materia, presentadas en tempo real.

Na Universidade Sénior, participaron 30 estudantes que compartiron a súas percepcións sobre diferentes temáticas como saúde, benestar e envellecemento e manifestaron coñecementos acadados sobre 0 uso das TIC.

- AnswerGarden é unha ferramenta online, gratuíta, que permite a creación dunha nube de palabras a partir dunha pregunta ou sentencia. Pode empregarse na aula coma un recurso educativo ou creativo para o intercambio de ideas ou brainstorming. Non precisa rexistro previo, pero permite igualmente moderar a presentación dos resultados e a participación do estudantado. Deste xeito, ofrece unha síntese visual e rápida das percepcións e/ou coñecementos do alumnado sobre un tema concreto. A pregunta URL da páxina pode inserirse nunha presentación ou abrirse cun navegador, indicando aos participantes un código numérico para 0 acceso. (Answergarden.ch, 2018).

- Mentimeter: É unha ferramenta interactiva que, a partir de deseños predefinidos, permite crear unha sucesión de enquisas, nubes de palabras, preguntas ou pantallas de información, que son presentadas en liña ou diferido, accedendo as/os participantes ás preguntas preparadas cos seus dispositivos tecnolóxicos. Unha vez creada a presentación, o docente pode compartila co estudantado, indicando unha dirección URL (menti.com) e ofrecendo un código numérico co que poder acceder á actividade online (Mentimeter, 2018).

- Kahoot é unha aplicación web que amosa "taboleiros de xogo" creados polo propio docente, a partir de deseños predefinidos ou desde una páxina en branco. As posibles propostas de actividades que permite presentar Kahoot son: quiz, xogos, discusión e 
enquisa. Para acceder ao "xogo" previamente deseñado polo docente, os estudantes ou xogadores introducen un código numérico (de xeito similar ás ferramentas anteriores). Ao rematar a sesión, aparece o listado de participantes, destacando as persoas que acadaron as puntuacións máis elevadas. 0 docente pode exportar os datos de participación a unha folla de cálculo (Kahoot!, 2018).

Os tres recursos explicados facilitan a monitorización da participación e asistencia dos estudantes na sesión, así coma o rexistro das respostas ás cuestións propostas, axudando no proceso de avaliación. Ademais, ofrecen versións para aplicar na docencia e nos espazos educativos, con maiores prestacións e posibilidades de rexistro.

\section{RESULTADOS}

Na Táboa 1 amósanse os principais resultados e características das aplicacións que conforma esta experiencia.

\begin{tabular}{|c|c|c|}
\hline & Grao en Terapia Ocupacional & Universidade Sénior \\
\hline $\mathrm{N}^{0}$ de participantes & $\begin{array}{c}105 \\
2^{0} \text { curso: } 56 \\
3^{0} \text { curso: } 48\end{array}$ & 30 \\
\hline Materias $^{i}$ & $\begin{array}{c}\text { Métodos informáticos para persoas con } \\
\text { discapacidade (TIC) } \\
\text { Terapia Ocupacional (TO) para a autonomía } \\
\text { persoal na infancia e adolescencia (Infancia) } \\
\text { T0 para a autonomía persoal no adulto con } \\
\text { discapacidade física (Adulto I) } \\
\text { T0 para a autonomía persoal no adulto con } \\
\text { disfunción na saúde mental (Adulto II) } \\
\text { TO para a autonomía persoal nas persoas } \\
\text { maiores (Maiores) } \\
\end{array}$ & $\begin{array}{l}\text { Estimulación cognitiva a } \\
\text { través das TIC (optativa) - } \\
\text { Ferramentas tecnolóxicas } \\
\text { para apoiar a saúde e a } \\
\text { vida activa }\end{array}$ \\
\hline $\begin{array}{l}\text { Ferramentas } \\
\text { empregadas }\end{array}$ & $\begin{array}{c}\text { TIC: Kahoot } \\
\text { Infancia: Kahoot } \\
\text { Adulto I: AnswerGarden } \\
\text { Adulto II: AnswerGarden } \\
\text { Maiores: AnswerGarden }\end{array}$ & $\begin{array}{c}\text { AnswerGarden, Kahoot e } \\
\text { Mentimeter }\end{array}$ \\
\hline Idades & $19-25$ anos & Máis de 55 anos \\
\hline
\end{tabular}

Táboa 1: Resultados da aplicación da gamificación no Grao en Terapia Ocupacional e na Universidade Senior 
Como resultados visuais amósanse varias figuras que evidencian resultados das diferentes ferramentas empregadas (Imaxe 1, Imaxe 2, Imaxe 3, Imaxe 4).

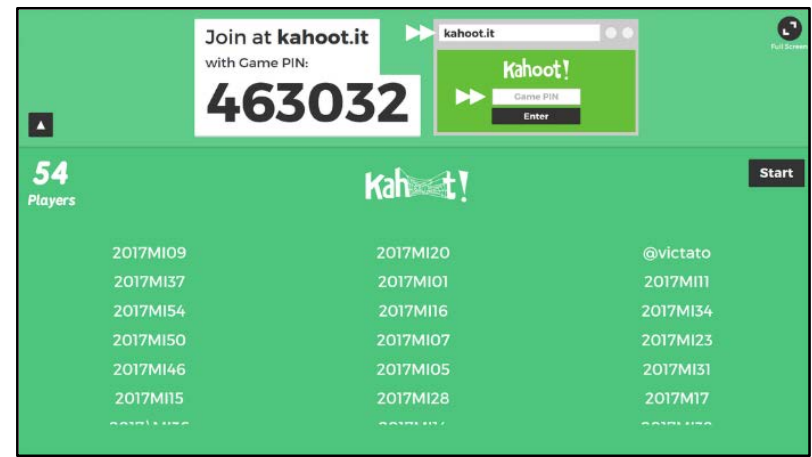

Imaxe 1: Invitación ao alumnado de TIC a participar en Kahoot

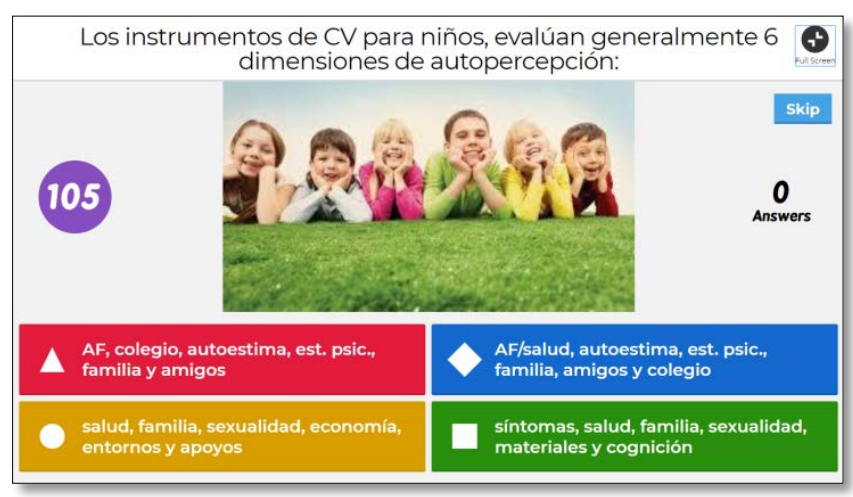

Imaxe 2: Resultados do emprego de AnswerGarden na materia de Infancia

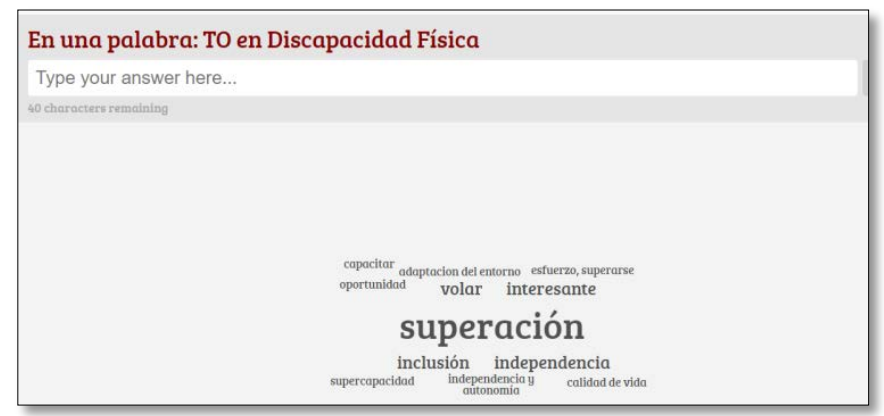

Imaxe 3: Resultados do emprego de AnswerGarden na materia de Adulto I 


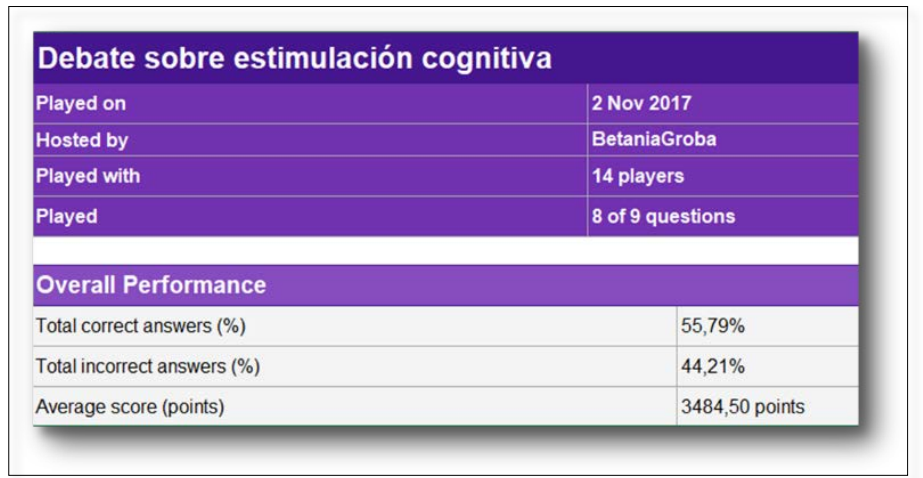

Imaxe 4: Cuantificación dos resultados de participación no Kahoot, aplicada na Universidade Senior

Nesta experiencia observouse unha maior motivación do alumnado para participar activamente nas clases.

Ademais, a variabilidade das opcións de presentación de contidos e interacción que permiten estas ferramentas pode ser un elemento de importante dinamización, xa que se xeran expectativas altas nos estudantes e se fomenta a súa implicación no propio proceso de aprendizaxe.

Unha das principais aportacións desta experiencia é o uso da gamificación cun grupo de persoas maiores (Universidade Sénior), e a súa comparación coa participación dinámica dun grupo de estudantes menores de 25 anos. A pesares da diferenza de idade e da posible brecha dixital entre os grupos, a motivación e a participación durante as clases foron moi similares. Finalmente, outros beneficios obtidos e derivados da experiencia son a facilitación da aprendizaxe de contidos específicos, o aumento da participación activa e dinamización da aula, e a satisfacción académica por obter unha retroalimentación inmediata.

Pola contra, os docentes atopáronse con certas limitacións durante 0 emprego destas ferramentas, como as dificultades de conectividade á rede wifi e datos $4 G$, xa que nalgunhas das aulas empregadas a cobertura é escasa, ou a dispoñibilidade de dispositivos móbiles suficientes para garantir a participación de todo 0 alumnado. Tamén constátase que a falta de 
accesibilidade e usabilidade das TIC pode limitar o seu uso por parte de certos grupos da poboación.

Tendo en conta esta e outras experiencias sobre a gamificación nas aulas do ensino superior, poden establecerse certas recomendacións xerais para potenciar a aprendizaxe dos estudantes en espazos tecnolóxicos:

- Crear dous tipos de contas: un coma docente e outra coma participante

- Ofrecer vídeos instrutivos sobre aspectos técnicos relacionados co acceso e manexo das plataformas ou recursos empregados.

- Proporcionar instrucións claras e sinxelas, acompañadas se é posible de imaxes e exemplos representativos.

- Amosar correccións xerais dos exercicios en vídeo.

- En plataformas para videoconferencias, recoméndase realizar unha proba inicial de conexión por separado con cada estudante.

- Ofrecer manuais e guías de apoio, especialmente para estudantes que se inicien no uso das TIC.

\section{CONCLUSIÓNS}

De xeito xeral, pódese indicar que os membros do grupo consideran estimulante a aplicación destas novas metodoloxías, tanto para o docente como para as/os estudantes.

A gamificación xorde para quedarse nas aulas universitarias, pero é preciso reflexionar sobre unha boa práctica: 0 docente ten a responsabilidade de definir claramente os obxectivos pretendidos co uso dos elementos lúdicos e de articulalos de tal xeito que teñan un sentido na presentación das clases e permitan abordar uns contidos mínimos da materia, captando a atención do alumnado (Contreras Espinosa \& Eguia, 2016).

Deste xeito, o equipo docente ten que valorar non só os recursos dispoñibles na aula, senón tamén as capacidades dos participantes, os seus coñecementos previos, así coma velar 
porque a integración da proposta de gamificación sexa coherente e non desvirtúe a adquisición das competencias e o núcleo central da materia.

Nesta primeira experiencia coas enquisas online, comprobouse que a gamificación pode ser aplicada nos contornos universitarios con diferentes grupos de estudantes. 0 feito de que a participación e visualización dos resultados se produza en tempo real, contribúe á participación activa de todos os implicados, incluídos os docentes, o mantemento da atención e motivación, e a retroalimentación inmediata, e apoia á aprendizaxe de contidos específicos das materias.

Proponse, para próximos cursos, a optimización da aplicación destas ferramentas e 0 aumento da variabilidade das propostas, introducindo estratexias colaborativas online en tempo real. Tamén será interesante cuantificar e comparar o nivel de participación activa co emprego destas aplicacións entre materias.

\section{REFERENCIAS}

Answergarden.ch. (2018). AnswerGarden -Plant a Question, Grow Answers! Generate a live word cloud with your audience. Retrieved December 8, 2018 from https://answergarden.ch/

Barbosa, A., \& Soto, J. G. (2016). Gamification Jam Galicia. Difusión de la gamificación y formación del profesorado. In A. R. Arias \& M. A. Melcón Álvarez (Eds.), II Congreso Virtual Iberoamericano sobre Recursos Educativos Innovadores CIREI La diversidad funcional visual y la creatividad desde el paradigma psicológico (pp. 250-257). Alcalá de Henares: Universidad de Alcalá. Retrieved from https://www.researchgate.net/publication/320895925

Contreras Espinosa, J. L., \& Eguia, R. S. (2016). Gamificación en aulas unviersitarias. (IncCOM, Ed.). Barcelona: InCOM. Universitat Autonoma de Barcelona. Retrieved from http://incom.uab.cat

Deterding, S., Khaled, R., Nacke, L. E., \& Dixon, D. (2011). Gamification: Toward a Definition. Retrieved from http://gamification-research.org/wp-content/uploads/2011/04/02-Deterding- 


\section{Khaled-Nacke-Dixon.pdf}

Kahoot! (n.d.). Kahoot! | Learning Games | Make Learning Awesome! Retrieved December 8, 2018, from https://kahoot.com/

Mentimeter. (2018). Interactive presentation software - Mentimeter. Retrieved December 8, 2018, from https://www.mentimeter.com/

Parente, D. (2016). Gamificación en la educación. In R. Contreras \& J. Eguia (Eds.), Gamificación en las aulas universitarias (pp. 10-24). Barcelona: Universitat Autónoma de Barcelona.

Pérez, C., Carlos, J., García, C., Piqueras, M., Collado, V., del Dedo, D., Martín, P. (2011). Gamificación y Docencia: Lo que la Universidad tiene que aprender de los Videojuegos. In VIII Jornadas Internacionales de Innovación Universitaria Retos y oportunidades del desarrollo de los nuevos títulos en educación superior. Madrid. Disponible en: http://www.esp.uem.es/gsi/http://observatoriovideojuegos.wordpress.com/

Universidade da Coruña. (2018). Estudar Grao en Terapia Ocupacional (2018/2019). Retrieved December 8, 2018, Disponible en: http://estudos.udc.es/g//study/start/653G01V01 Universidade da Coruña. (2018). Universidade Senior. Plano de estudos. Acceso December 8, 2018, Disponible en: https://www.udc.es/gl/senior/plano_estudos/

'A fin de mellorar a comprensión das materias e non duplicar o nome das mesmas, establécense as seguintes abreviaturas para referirnos ás mesmas:

- $\quad$ TIC: Métodos informáticos para persoas con discapacidade

- Infancia: Terapia Ocupacional para a autonomía persoal na infancia e adolescencia

- Adulto I: Terapia Ocupacional para a autonomía persoal no adulto con discapacidade física

- Adulto II: Terapia Ocupacional para a autonomía persoal no adulto con disfunción na saúde mental

- Maiores: Terapia Ocupacional para a autonomía persoal nas persoas maiores 
